\title{
Loss-of-function mutations in the mtr efflux system of Neisseria gonorrhoeae
}

\author{
Wendy L. Veal, ${ }^{1}$ Ansley Yellen, ${ }^{1}$ Jacqueline T. Balthazar, ${ }^{1,3}$ Wubin Pan, ${ }^{2} \dagger$ \\ Brian G. Spratt ${ }^{2} \ddagger$ and William M. Shafer ${ }^{1,3}$
}

Author for correspondence: William M. Shafer. Tel: +1 404728 7688. Fax: +1 4043292210.

e-mail: wshafer@emory.edu

\author{
1 Department of \\ Microbiology and \\ Immunology, Emory \\ University School of \\ Medicine, Atlanta, \\ GA 30322, USA \\ 2 Microbial Genetics Group, \\ School of Biological \\ Sciences, University of \\ Sussex, Falmer, Brighton \\ BN1 9QG, UK \\ 3 Laboratories of Microbial \\ Pathogenesis, Medical \\ Research Service, VA \\ Medical Center (Atlanta), \\ Decatur, GA 30033, USA
}

\begin{abstract}
Resistance of Neisseria gonorrhoeae to antimicrobial hydrophobic agents (HAs) has been ascribed to the mtr (multiple transferable resistance) operon. This operon is composed of the $m$ tr $R$ gene, which encodes a transcriptional repressor (MtrR), and a three-gene complex (mtrCDE), which encodes cell envelope proteins (MtrC-MtrD-MtrE) that form an energy-dependent efflux pump. HA-hypersusceptible strains are often isolated from patients, but the genetic basis for such hypersusceptibility was heretofore unknown. The genetic basis of HA hypersusceptibility in laboratory-derived strains BR54 and BR87 was studied to learn if this trait could be linked to mutations in the mtr operon. Mutations in the mtrR gene of these strains that could be phenotypically suppressed by mutations in their mtrc or mtrD genes were identified. Thus, small deletions (4-10 bp) in the mtrC or mtrD genes of strains BR87 and BR54 that would result in the production of truncated efflux pump proteins that serve as a membrane fusion protein (MtrC) or transporter of HAs (MtrD) were found to be responsible for their HA-hypersusceptible property.
\end{abstract}

Keywords: gonococci, efflux pump, antibiotic hypersusceptibility

\section{INTRODUCTION}

Following the introduction of penicillin to treat gonorrhoea, strains of Neisseria gonorrhoeae expressing clinically significant levels of penicillin resistance emerged. Gonococcal clinical isolates displaying hypersusceptibility to penicillin and structurally diverse hydrophobic agents (HAs) such as drugs, dyes, detergents and host-derived compounds (fatty acids and bile salts) also arose at nearly the same time as penicillinresistant strains appeared. In one particular study, conducted in North Carolina in 1978, antibiotic-hypersusceptible strains represented upwards of $15 \%$ of all gonococcal clinical isolates (Eisenstein \& Sparling, 1978). The antibiotic-hypersusceptibility profiles of

\footnotetext{
tPresent address: TerraGen Diversity Inc., University of British Columbia, Vancouver, Canada V6T 123.

¥Present address: Wellcome Trust Centre for the Epidemiology of Infectious Diseases, Department of Zoology, Oxford University, South Parks Road, Oxford OX1 3PS, UK.

Abbreviations: HA, hydrophobic agent; MFP, membrane fusion protein; TX-100, Triton X-100.

The GenBank accession number for the sequence of the $\mathrm{mtr} C$ gene of strain BR87 reported in this paper is AF037041 and that of the mtrD gene of strain
} BR54 is AF037040. these clinical isolates were remarkably similar to a set of laboratory-derived mutants and transformants that contained mutations in different loci termed env (envelope) (Sarubbi et al., 1975). On the basis of recombination studies (Eisenstein \& Sparling, 1978; Shafer et al., 1984), these antibiotic-hypersusceptible clinical isolates appeared to contain mutations in one of three different env loci, but most env mutations appeared to map at or near the $e n v-2$ locus of the laboratoryderived HA-hypersusceptible strain BR87. Although Env $^{-}$mutants and transformant strains were found to be hypersusceptible to structurally diverse HAs, many also contained a phenotypically suppressed mutation, now known to be in the $m t r R$ gene (Hagman et al., 1995), which could confer HA resistance when introduced into wild-type strain FA19 (Maness \& Sparling, 1973; Sarubbi et al., 1975; Shafer et al., 1984). The biochemical basis for the Env ${ }^{-}$property of such mutants has not been resolved, although earlier studies suggested that such strains had decreased amounts of a $52 \mathrm{kDa}$ outermembrane protein and decreased cross-linking of their peptidoglycan (Guymon et al., 1978). These biochemical changes in the cell envelope chemistry of Env ${ }^{-}$strains were postulated to be responsible for their enhanced permeability to HAs.

The $m t r$ locus in N. gonorrboeae (Maness \& Sparling, 
Table 1. Properties of HA resistant and susceptible isogenic strains

\begin{tabular}{|c|c|c|c|c|c|}
\hline \multirow[t]{2}{*}{ Strain } & \multirow[t]{2}{*}{ Genotype } & \multirow[t]{2}{*}{ Source } & \multicolumn{3}{|c|}{$\operatorname{MIC}\left(\mu \mathrm{g} \mathrm{ml}^{-1}\right)^{*}$} \\
\hline & & & Ery & PenG & TX-100 \\
\hline FA19 & Wild-type & P. F. Sparling & $0 \cdot 25$ & 0.007 & 100 \\
\hline FA140 & penA penB mtrR-140 & P. F. Sparling & $2 \cdot 00$ & $1 \cdot 000$ & $>16000$ \\
\hline BR54 & penA penB mtrR-140 mtrD-54 (env-3)† & P. F. Sparling & $0 \cdot 06$ & 0.060 & 25 \\
\hline BR87 & penA penB mtrR-87 mtrC-87 (env-2)† & P. F. Sparling & 0.06 & 0.060 & 25 \\
\hline WV1 & As BR54 but $m t r D^{+}$ & FA19 $¥ \times$ BR54 & $2 \cdot 00$ & $1 \cdot 000$ & $>16000$ \\
\hline WV2 & As BR54 but $m t r D^{+}$ & Spontaneous mutant & $1 \cdot 00$ & 0.500 & $>16000$ \\
\hline WV3 & As BR54 but $m t r D^{+}$ & WV $2 \ddagger \times$ BR54 & $1 \cdot 00$ & 0.500 & $>16000$ \\
\hline AY1 & As BR87 but $m t r C^{+}$ & FA19 $¥ \times$ BR87 & $1 \cdot 00$ & $0 \cdot 125$ & $>16000$ \\
\hline
\end{tabular}

* Ery, erythromycin; PenG, penicillin G.

†Former designations (Sarubbi et al., 1975) for the $m t r D-54$ (env-3) and $m t r C-87$ (env-2) genes of strains BR54 and BR87, respectively, are in parentheses.

¥ Transformant strain WV1 was obtained using the 964 bp PCR product from the $m$ trD gene of strain FA19 (Fig. 1), transformant WV3 was obtained using the same 964 bp PCR product from WV2 and transformant AY1 was obtained using the PCR-amplified $m t r C$ gene from strain FA19.

1973; Guymon \& Sparling, 1975) has been recently characterized (Pan \& Spratt, 1994; Hagman et al., 1995) and consists of the mtrR gene, positioned $250 \mathrm{bp}$ upstream and divergently transcribed from a three-gene (mtrCDE) complex (Fig. 1) encoding cell envelope proteins that form an energy-dependent efflux pump (Lucas et al., 1995). This efflux pump, not altered cell envelope permeability (Guymon \& Sparling, 1975), appears to be responsible for the HA-resistant property exhibited by certain strains of gonococci (Pan \& Spratt, 1994; Shafer et al., 1995). Moreover, the mtr efflux system is crucial for expression of maximal levels of chromosomally mediated penicillin resistance that results from mutations at unlinked genes (Sparling et al., 1975).

The MtrR protein appears to be a transcriptional repressor and performs a critical role in regulating transcription of the $m t r C D E$ gene complex (Hagman et al., 1995). Thus, mutations within the mtrR-coding region or a single base pair deletion in a 13 bp inverted repeat in its promoter enhance $m t r C D E$ gene expression leading to elevated HA resistance (Hagman et al., 1995). However, overexpression of the mtrCDE gene complex has a physiological consequence since it is known (Eisenstein \& Sparling, 1978) that transformant strains bearing $m t r R$ mutations display a slower growth rate than that of the wild-type parental strain. A possible selective advantage in vivo, in the absence of HAs (or penicillin), for strains bearing $e n v$ mutations is that the reduced growth rate imparted by mutations in $m t r R$ can be reversed to wild-type levels by a co-resident mutation in $e n v-2$ or $e n v-3$ (Eisenstein \& Sparling, 1978).

Based on the deduced amino acid sequences of the MtrC-MtrD-MtrE proteins, it is clear that the $m t r$ efflux pump in gonococci is similar to the MexAMexB-OprM pump of Pseudomonas aeruginosa and the AcrA/E-AcrB/F-TolC systems of Escherichia coli (reviewed by Nikaido, 1994, 1996; Paulsen et al., 1996). The gonococcal MtrC protein belongs to the membrane fusion protein (MFP) family (Saier et al., 1994) that links the cytoplasmic-membrane MtrD transporter protein, which belongs (Hagman et al., 1997) to the resistance/ nodulation/division protein family (Saier et al., 1994), to the MtrE outer-membrane protein. The MtrE protein probably forms an outer-membrane channel that facilitates export of HAs (Delahay et al., 1997), in a manner proposed for the TolC and OprM outer-membrane proteins of $E$. coli and $P$. aeruginosa, respectively (Nikaido, 1996). As the mtrCDE-encoded efflux pump performs a predominant role in determining levels of gonococcal resistance to HAs, including toxic fatty acids and bile salts that bathe certain mucosal surfaces (Morse et al., 1982), we analysed whether the $\mathrm{Env}^{-}$strains studied previously by Sarubbi et al. (1975) contain mutations in their mtrCDE gene complex. We now report that these $\mathrm{Env}^{-}$strains contain small deletions in their $m t r C$ or $m t r D$ genes and that these mutations are responsible for their HA-hypersusceptible trait.

\section{METHODS}

Strains of $\boldsymbol{N}$. gonorrhoeae employed and growth conditions. Strain FA19 and a panel of isogenic transformant strains (Table 1 ) were kindly provided by P. F. Sparling (University of North Carolina, Chapel Hill, NC, USA). HA hypersusceptibility was defined when the MIC of erythromycin was $<0.12 \mu \mathrm{g} \mathrm{ml}^{-1}$ and that of Triton X-100 (TX-100) was $<62 \mu \mathrm{g} \mathrm{ml}^{-1}$. All strains were propagated on GCB agar (Difco) containing glucose and iron supplements (Shafer et al., 1984) with incubation at $37^{\circ} \mathrm{C}$ under $3.8 \%(\mathrm{v} / \mathrm{v}) \mathrm{CO}_{2}$.

PCR amplification and DNA sequencing. Chromosomal DNA was prepared by the method of McAllister \& Stephens (1993) and used in PCR amplification reactions as described pre- 
Table 2. Oligonucleotides used to amplify $m$ trRCDE genes

\begin{tabular}{|c|c|c|c|}
\hline $\begin{array}{l}\text { Gene } \\
\text { amplified }\end{array}$ & Oligonucleotide & Sequence & Site \\
\hline \multirow[t]{2}{*}{$m t r R$} & KH9\#3 & $5^{\prime}-$ GACGACAGTGCCAATGCAACG-3' & $\begin{array}{l}47 \mathrm{nt} \text { downstream of } m t r C \text { start; C } \\
\text { strand of } m t r R\end{array}$ \\
\hline & CEL\#1 & $5^{\prime}-$ GACAATGTTCATGCGATGATAGG $-3^{\prime}$ & $\begin{array}{l}114 \mathrm{nt} \text { downstream of } m t r R \text { stop; NC } \\
\text { strand of } m t r R\end{array}$ \\
\hline \multirow[t]{3}{*}{$m t r C$} & KH9\#1 & 5'-GTCGCAGATACGTTGGAACAACG-3' & $\begin{array}{l}174 \mathrm{nt} \text { downstream of } m t r R \text { start; C } \\
\text { strand of } m t r C\end{array}$ \\
\hline & $m t r D \# 2$ & 5'-GCCGTACCCAACTGCCCTTGC- $3^{\prime}$ & $\begin{array}{l}702 \mathrm{nt} \text { downstream of } m t r D \text { start; NC } \\
\text { strand of } m t r C\end{array}$ \\
\hline & KH9\#4 & $5^{\prime}$-CATCACTTGTGCGGACGCACC-3' & $\begin{array}{l}151 \mathrm{nt} \text { downstream of } m t r D \text { start; NC } \\
\text { strand of } m t r C\end{array}$ \\
\hline \multirow[t]{4}{*}{$m t r D$} & $m t r D \# 1$ & 5'-CGGCATCTGAAGCCAAACCTGC-3' & $\begin{array}{l}10 \mathrm{nt} \text { upstream of } m t r C \text { stop; C } \\
\text { strand of } m t r D\end{array}$ \\
\hline & $m t r E \# 1$ & $5^{\prime}$-GATGGAAGAAACCGATGTGTCG- $3^{\prime}$ & $\begin{array}{l}120 \mathrm{nt} \text { downstream of } m t r E \text { start; NC } \\
\text { strand of } m t r D\end{array}$ \\
\hline & $m t r D \# 10$ & $5^{\prime}$-AGCATCAACCTGCAAGACCGC- $3^{\prime}$ & $\begin{array}{l}2028 \mathrm{nt} \text { downstream of } m t r D \text { start; C } \\
\text { strand of } m t r D\end{array}$ \\
\hline & $m t r D \# 7$ & $5^{\prime}$-ATATACAGGGGAACCACGCCC- $3^{\prime}$ & $\begin{array}{l}208 \mathrm{nt} \text { upstream of } m t r D \text { stop; NC } \\
\text { strand of } m t r D\end{array}$ \\
\hline \multirow[t]{2}{*}{$m t r E$} & $m t r E \# 2$ & 5'-CCTTTGCATTGTCTGCCTGCAC-3' & $\begin{array}{l}43 \mathrm{nt} \text { downstream of } m t r E \text { start; C } \\
\text { strand of } m t r E\end{array}$ \\
\hline & $m t r E \# 3$ & 5'-CGGTTTGGGTATCCGTTTCAATC-3' & $\begin{array}{l}5 \mathrm{nt} \text { upstream of } m t r E \text { stop; NC } \\
\text { strand of } m t r E\end{array}$ \\
\hline
\end{tabular}

* Sites of annealing of oligonucleotides at nucleotide positions are shown upstream or downstream from translational start or stop codons for $m t r R C D E$ genes that are on coding (C) or non-coding (NC) strands.

viously (Hagman et al., 1995). The oligonucleotide primers used in this investigation are described in Table 2. DNA sequencing was performed on PCR products using the cycle sequencing protocol (Hagman et al., 1995) or by automatic DNA sequencing; automatic sequencing was performed by the Nucleic Acid Core Facility of Emory University. Nucleotide and amino acid sequence analysis was performed using DNAStar.

Transformation and isolation of spontaneous mutants. Piliated gonococci were transformed with bulk chromosomal DNA or PCR products prepared from strain FA19 essentially as described by Sarubbi et al. (1975). Transformation experiments initially employed PCR products immediately after the amplification reaction. In subsequent experiments, products with transforming activity were purified by agarose gel electrophoresis to exclude transformation due to the input template DNA. Erythromycin-resistant transformants of strains BR54 and BR87 were selected using erythromycin at 0.12 or $0.25 \mu \mathrm{g} \mathrm{ml}^{-1}$. Transformants were scored for levels of resistance to erythromycin, penicillin $G$ and $T X-100$ as described previously (Shafer et al., 1984). Spontaneous HAresistant mutants were selected by plating suspensions onto GCB agar containing erythromycin $\left(0.12\right.$ or $\left.0.25 \mu \mathrm{g} \mathrm{ml}^{-1}\right)$ or TX-100 (500 $\left.\mu \mathrm{g} \mathrm{ml}^{-1}\right)$.

SDSPAGE and Western blotting. Whole-cell lysates of gonococcal strains were prepared as described previously (Hagman et al., 1995), while total cell envelope proteins were prepared as described by Clark et al. (1987). Proteins were separated by SDS-PAGE (Laemmli, 1970) and electroblotted to nylon membranes (Hagman et al., 1995). MtrE was detected using an anti-MtrE mouse antiserum (kindly provided by $\mathrm{C}$. Ison, $\mathrm{St}$
Mary's Hospital Medical School, London, UK) and goat antimouse IgG conjugated to alkaline phosphatase.

\section{RESULTS AND DISCUSSION}

\section{Presence of phenotypically suppressed $m$ trR mutations in HA-hypersusceptible transformant strains}

HA-hypersusceptible strains BR54 (env-3) and BR87 $(e n v-2)$ were generated in P. F. Sparling's laboratory in the mid-1970s (Sarubbi et al., 1975) using donor DNA from HA-hypersusceptible strains FA32 (for BR54) or FA47 (for BR87) to transform HA-resistant strain FA140. The $e n v-3$ and $e n v-2$ mutations in strains BR54 and BR87 were postulated to occur at different sites because they recombine frequently, yielding HA-resistant transformants (Sarubbi et al., 1975; Eisenstein \& Sparling, 1978; Shafer et al., 1984). Sarubbi et al. (1975) found that strains BR54 and BR87 could also donate high-level HA resistance to wild-type strain FA19 in these transformation experiments, suggesting that they also contain a phenotypically suppressed mutation in their $m t r R$ gene (Pan \& Spratt, 1994). In support of this hypothesis, we previously found (Hagman et al., 1995) that the $m t r R$ gene of strain BR87 differed from that of strain FA19 by a missense mutation at codon 45 (GGC to $\mathrm{G} \underline{\mathrm{A}}$ ), which resulted in the replacement of the glycine residue with an aspartic acid at position 45 , which is within the helix-turn-helix region of the MtrR 

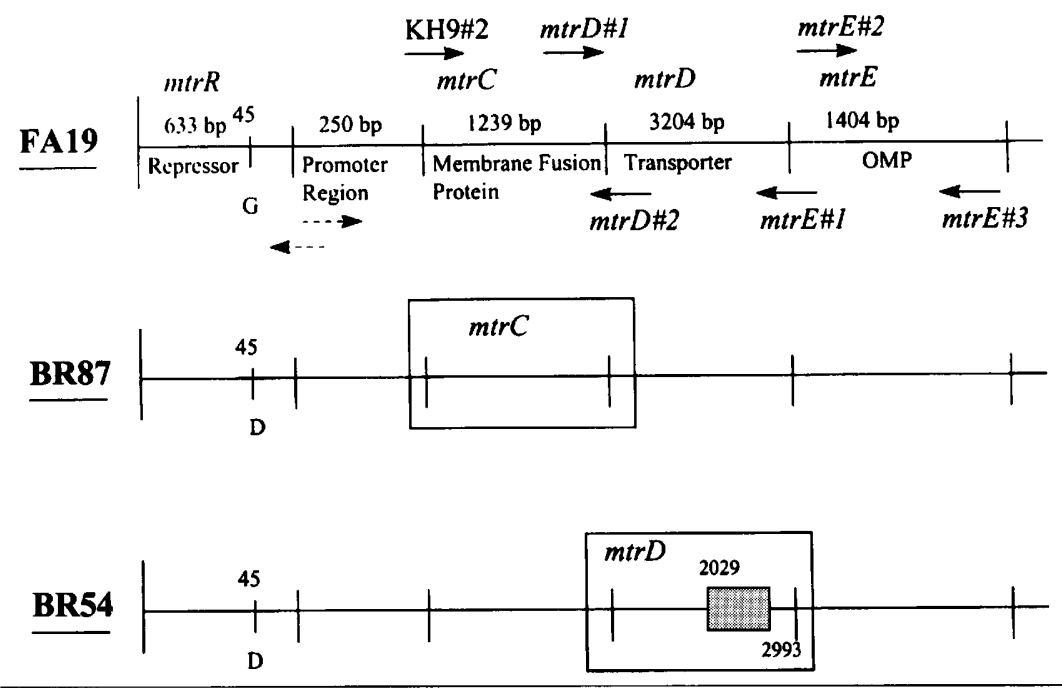

Fig. 1. Organization of the mtr efflux operon of $N$. gonorrhoeae strain FA19. On the line marked FA19, the gene products and their function, the size (not drawn to scale) in bp of each gene and the approximate sites of annealing and direction of oligonucleotide primers used to amplify the mtrCDE genes from strain FA19 for transformation experiments are described. The arrows with dashed lines underneath the promoter region show the direction of transcription of the $m t r R$ and $m$ trCDE genes (Hagman \& Shafer, 1995). The glycine residue at position 45 of the MtrR protein produced by strain FA19 is shown (G). The glycine-45 to aspartic acid (D) substitution within the MtrR protein produced by strains BR87 and BR54 is shown. In addition to this missense mutation, strain BR54 also contains a single base pair deletion within the $13 \mathrm{bp}$ inverted repeat localized within the $m$ trR promoter (data not presented). The regions of the $\mathrm{mtr}$ operon of strain FA19 capable of transforming strains BR87 and BR54 for HA resistance are shown in the boxed regions on their respective lines. The darkened box within the mtrD gene of strain BR54 shows the region (nucleotide positions 2029-2993) mapped by transformation that contained the $10 \mathrm{bp}$ deletion responsible for HA hypersusceptibility in this strain.

repressor (Pan \& Spratt, 1994). This radical amino acid substitution abrogates binding of the MtrR repressor to its target DNA sequence, the overlapping, divergent promoters used for $m t r R$ and $m t r C D E$ expression (Lucas et al., 1997). Importantly, when introduced into strain FA19 by transformation, this missense mutation provided for increased HA resistance (Hagman et al., 1995).

To determine the nature of the phenotypically suppressed $m t r R$ gene in strain BR54, we sequenced its $m t r R$ gene and that of HA-resistant parental strain FA140. Using oligonucleotide primers previously employed (Table 2; Shafer $e$ t al., 1995) to amplify the $m t r R$ gene, we determined that the $m t r R$ gene from strains BR54 and FA140 also contained the missense mutation in codon 45 present in BR87 (Fig. 1) but, unlike BR87, they also contained a single base pair deletion in the $13 \mathrm{bp}$ inverted repeat within the promoter utilized for $m t r R$ gene transcription (Hagman \& Shafer, 1995). This base pair deletion in the $m t r R$ promoter abrogates transcription of $m t r R$ but enhances transcription from the overlapping, divergent promoter used for $m \operatorname{tr} C D E$ gene transcription (Hagman \& Shafer, 1995) and is sufficient to provide high-level HA resistance (Hagman et al., 1995; Shafer et al., 1995).

\section{Strains BR87 and BR54 contain small deletions in their $\boldsymbol{m t r} C$ and $\boldsymbol{m t r} D$ genes, respectively}

Since the HA-susceptibility profiles of strains BR54 and BR87 (Sarubbi et al., 1975 and Table 1) resembled those of mutants of strain FA19 that contained insertionally inactivated $m$ trCDE genes (Hagman et al., 1995, 1997; Delahay et al., 1997), we hypothesized that the $e n v-2$ and $e n v-3$ mutations might reside within the mtrCDE gene complex. To test this hypothesis, the $m t r C, m t r D$ and $m t r E$ genes from wild-type strain FA19 were amplified by PCR and used to transform strains BR54 and $\mathrm{BR} 87$ for HA resistance.

With strain BR87 (env-2) as the recipient, only the $m t r C$ encoding sequence (obtained with oligonucleotide primers KH9\#1 and $m t r D \# 2$; Table 2) from strain FA19 exhibited transforming activity (Fig. 1). A representative transformant (strain AY1) displayed enhanced resistance to TX-100 and a twofold increase in penicillin resistance. DNA sequencing of the $m t r C$ gene from strains BR87, FA140 and AY1 was undertaken to identify the mutation(s) that might be responsible for HA hypersusceptibility in BR87. The results showed that compared to the $m t r C$ gene from strain FA19 (GenBank accession no. U14993; Hagman et al., 1995), the $m t r C$ gene of strain BR 87 contained a 4 bp deletion between nucleotide positions 349 and 352 and single nucleotide changes at codons 152 (GCG to GTG), 161 (GAA to GAG), 162 (GCA to GCG), 163 (AGC to GGC) and 390 (AAC to AAT) (Fig. 2). All of the single nucleotide changes, but not the 4 bp deletion, were also present in parental strain FA140 (data not presented). The 4 bp deletion represents, on the $m t r C$-coding strand, the last four nucleotides of a six dinucleotide (GC) repeat (341-GCGCGCGCGCGC-352; underline shows deletion in BR87) and generates a new stop codon (Fig. 2). Due to this frameshift, the MtrC protein produced by 


\begin{tabular}{|c|c|c|c|c|}
\hline & $340 \longrightarrow 360$ & $450-463$ & $480-490$ & $1167-$ \\
\hline FA19 & $\frac{\text { AGCGCGCGCGCGCAACTGGCA }}{\text { GC Repent }}$ & CGATGCTGCGGTAA & CGAAGCAAGCG & AAACCAAGCTGCCG \\
\hline BR87 & $\begin{array}{c}\text { AGCGCGCGC .... AACTGGCA } \\
\text { a bp Deletion }\end{array}$ & $\begin{array}{r}\text { CGATGCTGIGGTAA } \\
\text { Stop }\end{array}$ & CGAGGCGGGCG & AAATCAAGCTGCCG \\
\hline A) & AGCGCGCGCGCGCAACTGGCA & CGATGCTGCGGTAA & cGaAgcangcg & AAACCAAGCTGCCG \\
\hline
\end{tabular}

Fig. 2. Identification of the 4 bp deletion (dashed line) and point mutations (doubleunderlined nucleotides) in the mtrC gene of HA-hypersusceptible strain BR87 compared to the sequences possessed by strains FA19 and AY1. The four solid lines flanked by numbers at the top of the figure show the nucleotide positions of the relevant sequences in the mtrC gene. The new stop codon (TAA) in the BR87 sequence is shown with a single underline.

\begin{abstract}
FA19 GGT GCG GCT.GCG GGCGTA ACC GGG CGC ACC CTG TTT GAM GGA CTG TTG GGC 272
$\begin{array}{llllllllllllllllll}G & A & A & A & G & G & V & T & G & R & N & L & F & E & G & L & L & G\end{array}$ **

BR5A GGT GCG GCT GCG GGC GCA ACC TGT TTG AAG GAG TGT TGG GCA GCG TTC CCT G A A A G A T C L L K

W1 GGT GCG GCT GCG GGC GTAACC GGG CGC AAC CTO TTT GAM GGA CTO TTG GQC $G$ A A A G $V$ V T G

w2

GGT GCG GCTGCG GGC - - - - - aAC CTG TTT GMAGGA CTG TTG GOC

G A A A G N L F E G L L G

Fig. 3. Nucleotide sequence of a 51 nucleotide region (nucleotides 2721-2772) of the mtrD gene of strains FA19, BR54, $W V 1$ and $W V 2$. The 10 bp region that is deleted in strain BR54 is shown in the underlined stretch in the sequence of strains FA19 and HA-resistant transformant strain WV1. The encoded amino acids are shown in the single-letter code. The additional 2 bp that are deleted in spontaneous mutant WV2 are shown with asterisks in the BR54 sequence. The 12 bp deleted in WV2 are shown by dashed lines.
\end{abstract}

strain BR87 would be only 152 amino acids in length, diverging from the MtrC protein of strain FA19 (412 amino acids; molecular mass of $42777 \mathrm{Da}$ ) after amino acid position 116. The nucleotide sequence of the $m t r C$ gene from transformant strain AY1 (Table 1) was determined and the results showed that the 4 bp deletion (Fig. 2) and each of the six point mutations described above had been repaired so as to resemble the wild-type sequence. Since the MtrC lipoprotein is a member of the MFP family (Saier et al., 1994), it is likely that the severely truncated MtrC protein produced by BR87 is unable to interact with MtrD or MtrE or both, resulting in the loss of overall structural integrity of the $m t r$ efflux pump.

In contrast with the results obtained with BR87, only the $m t r D$ sequence (obtained with oligonucleotide primers $m t r D \# 1$ and $m t r E \# 1$; Table 2) from strain FA19 was able to transform strain BR54 for HA resistance (Fig. 1). To localize the region of the $3.2 \mathrm{~kb} m t r D$ gene in strain BR54 responsible for its HA-hypersusceptible property, a panel of oligonucleotide primers that would amplify overlapping regions of the $m t r D$ gene from strain FA19 (GenBank accession no. U60099) was used to generate PCR products for transformation. With these different PCR products, the transforming activity of the $m t r D$ sequence could be narrowed to a $964 \mathrm{bp}$ fragment (obtained with oligonucleotide primers $m t r D \# 10$ and $m t r D \# 7$; Table 2) located near the $3^{\prime}$-end of the gene (shown as nucleotide positions 2029-2993 in Fig. 1). A representative transformant (WV1) was as HA resistant as the immediate parental strain of BR54 (strain FA140; Table 1).

A mutation in the $m t r D$ gene from strain BR54 was identified by sequencing the aforementioned PCR product obtained from strains FA19, BR54 and transformant strain WV1. From this analysis it was determined that compared to strains FA19, FA140 and WV1, strain BR54 contained a $10 \mathrm{bp}$ deletion (5'-CGTAACCGGG-3'; nucleotide positions $2736-2745$ in the $m t r D$ gene of strain FA19), while WV1 had this deletion repaired (Fig. 3 ). The $10 \mathrm{bp}$ deletion would result in the production of an MtrD protein that would diverge from the wild-type sequence at amino acid position 912. Moreover, due to the introduction of a new stop codon (TGA; nucleotides 2825-2827 in the $m t r D$ gene of strain FA19), the MtrD protein produced by strain BR54 would contain 938 amino acids (molecular mass of $99935 \mathrm{Da}$ ) compared to the 1067 amino acid MtrD protein produced by strain FA19 (molecular mass of $113928 \mathrm{Da}$ ). Importantly, a topology model for the MtrD protein of strain FA19 has been deduced (Hagman et al., 1997) and it predicts the existence of 12 transmembrane domains, seven cytoplasmic loops and six periplasmic loops. From this model, the MtrD protein produced by BR 54 would lack the last three $\mathrm{C}$-terminal transmembrane domains as well as two periplasmic and cytoplasmic loops with the last 26 amino acids being located in the cytoplasm (M. Nilles, personal communication).

\section{Isolation of spontaneous HA-resistant mutants}

To identify mutations that might reverse the effects of the small deletions in the $m t r C$ and $m t r D$ genes in BR87 and BR54, respectively, spontaneous HA-resistant mutants of strains BR54 and BR87 were sought. Erythromycin-resistant mutants of strain BR54 arose at a low frequency $\left(4 \times 10^{-9}\right)$ but such mutants of BR87 could not be recovered (frequency $\left\langle 10^{-10}\right.$ ) despite repeated efforts. A spontaneous erythromycin-resistant mutant (WV2) of BR54 displayed high-level resistance to TX100 (MIC $>16000 \mu \mathrm{g} \mathrm{ml}^{-1}$ ) but resistance to erythromycin and penicillin was twofold lower than that of FA140 or WV1 (Table 1). The DNA sequence of the 
$m t r D$ gene from strain WV2 that could be amplified by PCR with primers $m t r D \# 10$ and $m t r D \# 7$ was determined. The results showed that an additional $2 \mathrm{bp}$ had been deleted in the $m t r D$ sequence of strain WV2 (shown in Fig. 3 with asterisks above the BR54 sequence). This 2 bp deletion in strain WV2 would restore the correct reading frame for the $m t r D$ gene but would also result in a loss of four amino acids (Val-Thr-Gly-Arg) prior to returning to the wild-type amino acid sequence (Fig. 3). The Thr-Gly-Arg sequence represents the first three amino acids of the fifth periplasmic loop predicted to exist for MtrD (Hagman et al., 1997), but the deletion of the Val-Thr-Gly-Arg peptide is not predicted to significantly alter the topology map of MtrD (M. Nilles, personal communication). We confirmed the importance of the $2 \mathrm{bp}$ deletion in the $m t r D$ gene sequence in WV2 by transformation. Using a PCR product containing this region, we were able to obtain transformants of strain BR54 expressing enhanced HA resistance (see WV3 in Table 1) similar to that observed with donor strain WV2.

Heretofore, all mutations that altered HA resistance in gonococci have mapped to the $m t r R$ regulatory gene (mutations that enhance resistance) or were laboratoryderived insertional mutations in the $m t r C D E$ gene complex (mutations that decrease HA resistance). Moreover, a survey of the literature suggests that all other mutations in the resistance/nodulation/division protein class of efflux pumps (Saier et al., 1994), of which the $m t r$ system is a member, that decrease efflux pump activity represent regulatory mutants or strains with purposeful, laboratory-derived insertional mutations. Our finding that HA-hypersusceptible transformant strains BR54 and BR87 contain small deletions in their $m t r C D$ genes is the first report, to our knowledge, of loss-of-function-type mutations in bacterial efflux pump genes.

The findings presented in this report clarify the mechanism by which strains BR54 and BR87, the first laboratory-derived HA-hypersusceptible strains isolated in the gonococcus (Sarubbi et al., 1975), exhibit HA hypersusceptibility. While it was originally thought (Guymon \& Sparling, 1975) that these strains had increased permeability for HAs, it is now clear that their respective $m t r$ efflux pumps are abnormal. Thus, the small deletions in the $m t r C$ or $m t r D$ genes in strains BR87 and BR54 would result in truncated, partially nonsensical MtrC or MtrD proteins. Since MtrC is in the family of MFPs (Saier et al., 1994) that are presumed to link the cytoplasmic-membrane efflux transporter protein to the outer-membrane protein that serves as a channel for exported antimicrobials (Nikaido, 1996), it is likely that this function is abolished in strain BR87. Truncation of the MtrD protein in strain BR54 could result in HA hypersusceptibility due to loss of its transporter capacity or an inability to interact with its cognate MFP protein (MtrC). We considered the possibility that the $10 \mathrm{bp}$ deletion in the $m t r D$ gene would impact expression of the $m t r E$ gene, which is immediately downstream and in the same transcriptional unit, but an anti-MtrE antiserum (Delahay et al., 1997) recognized this $50 \mathrm{kDa}$ MtrE protein in strains FA19, BR54, WV1 and WV2 (data not presented). Thus, the HA-hypersusceptible phenotype in strain BR54 seems to be due to an altered MtrD transporter protein and not loss or diminished expression of $m t r E$.

We also confirmed that HA-hypersusceptible strains BR54 and BR87 contain phenotypically suppressed mutations in their mtrR gene, which is consistent with the earlier prediction of Sarubbi et al. (1975). The mutations occur within the $m t r R$-coding sequence that determines the amino acid composition of the helixturn-helix domain of MtrR or in a $13 \mathrm{bp}$ inverted repeat sequence that lies between the -10 and -35 regions of the mtrR promoter. When transferred to wild-type strain FA19, any of these mutations are sufficient to increase HA resistance (Hagman et al., 1995; Shafer et al., 1995). We have evaluated two recent HA-hypersusceptible clinical isolates because the original report of Eisenstein \& Sparling (1978) suggested that such strains would have both phenotypically suppressed mutations in $m t r R$ and mutations similar to the $m t r C$ mutation in strain BR87. We found that these two recent clinical isolates contained a single missense mutation in their $m t r R$-coding region that mapped to codon 40 (GCC to ACC), causing a replacement of alanine with threonine in the helix-turn-helix region of the MtrR repressor; this replacement is sufficient to mediate increased HA resistance in gonococci (Shafer et al., 1995). Surprisingly, however, the genetic lesion for HA hypersusceptibility in these strains appears not to map within the mtrCDE complex (data not presented). Accordingly, these recent clinical isolates, as opposed to those studied in the 1970s (Eisenstein \& Sparling, 1978), could have mutations that affect alternative efflux pumps or permeability systems or unlinked control systems that modulate $m t r$ efflux activity. Nevertheless, the presence of phenotypically suppressed mtrR mutations in these strains and the presence of an unlinked mutation(s) that results in hypersusceptibility of gonococci to HAs is consistent with the earlier hypothesis (Shafer et al., 1984) that $m$ trR mutations are first selected when gonococci are introduced into an environment bathed in HAs (such as the rectum). Subsequent secondary mutations that reverse the effect of $m t r R$ mutations (HA resistance and slower growth rate) could arise when gonococci enter other sites that may be hostile for those slower growing gonococci harbouring mtrR mutations. The genetic basis for HA hypersusceptibility in these clinical isolates is now under investigation.

\section{ACKNOWLEDGEMENTS}

This work was supported by NIH grant AI-21150 (W.M.S.) and funds from the VA Medical Research Service (W.M.S.). W.M.S, is the recipient of an Associate Career Scientist Award from the VA Medical Research Service. W.V. was supported by NIH training grant T32 AI-07470. Work in the laboratory of B.G.S. was supported by the Medical Research Council and Wellcome Trust. W.P. acknowledges the support from the Sir Run Run Shaw Scholarship Fund. 
We thank L. Pucko for help in manuscript preparation, M. Nilles for help in understanding the topology of MtrD, the staff of the Emory University Microchemical Facility for preparing oligonucleotides, the DNA sequencing core facility for automatic DNA sequencing services, and D. Stephens for critical evaluation of the manuscript prior to submission. W.M.S. acknowledges the encouragement of P.F. Sparling for pursuing these studies.

\section{REFERENCES}

Clark, V. L., Campbell, L. A., Palermo, D. A., Evans, T. M. \& Klimpel, J. W. (1987). Induction and repression of outer membrane proteins by anaerobic growth of Neisseria gonorrhoeae. Infect Immun 55, 1359-1364.

Delahay, R. M., Robertson, B. D., Balthazar, J. T., Shafer, W. M. \& Ison, C. A. (1997). Involvement of the gonococcal MtrE protein in the resistance of Neisseria gonorrhoeae to toxic hydrophobic agents. Microbiology 143, 2127-2133.

Eisenstein, B. I. \& Sparling, P. F. (1978). Mutations to increased antibiotic sensitivity in naturally-occurring gonococci. Nature 271, 242-244.

Guymon, L. F. \& Sparling, P. F. (1975). Altered crystal violet permeability and lytic behavior in antibiotic-resistant and -sensitive strains of Neisseria gonorrhoeae. J Bacteriol 124, 757-763.

Guymon, L. F., Walstad, D. L. \& Sparling, P. F. (1978). Cell envelope alterations in antibiotic-sensitive and -resistant strains of Neisseria gonorrhoeae. J Bacteriol 136, 391-401.

Hagman, K. E. \& Shafer, W. M. (1995). Transcriptional control of the $m$ tr efflux system of Neisseria gonorrboeae. J Bacteriol 171, 4162-4165.

Hagman, K. E., Pan, W., Spratt, B. G., Balthazar, J. T., Judd, R. C. \& Shafer, W. M. (1995). Resistance of Neisseria gonorrboeae to antimicrobial hydrophobic agents is modulated by the $\operatorname{mtrRCDE}$ efflux system. Microbiology 141, 611-622.

Hagman, K. E., Lucas, C. E., Balthazar, J. T., Snyder, L., Nilles, M., Judd, R. C. \& Shafer, W. M. (1997). The MtrD protein of Neisseria gonorrhoeae is a member of the resistance/nodulation/division protein family constituting part of an efflux system. Microbiology $143,2117-2125$.

Laemmli, U. K. (1970). Cleavage of structural proteins during the assembly of the head of bacteriophage T4. Nature 227, 680-685.

Lucas, C. E., Hagman, K. E., Levin, J. C., Stein, D. C. \& Shafer, W. M. (1995). Importance of lipooligosaccharide structure in determining gonococcal resistance to hydrophobic antimicrobial agents resulting from the $m$ tr efflux system. Mol Microbiol 16, 1001-1009.

Lucas, C. E., Balthazar, J. T., Hagman, K. E. \& Shafer, W. M. (1997). The MtrR repressor binds the DNA sequence between the $m t r R$ and $m t r C$ genes of Neisseria gonorrboeae. J Bacteriol 179, 4123-4128.

McAllister, C. F. \& Stephens, D. S. (1993). Analysis in Neisseria meningitidis and other Neisseria species of genes homologous to the FKBP immunophilin family. Mol Microbiol 10, 13-24.

Maness, M. J. \& Sparling, P. F. (1973). Multiple antibiotic resistance due to a single mutation in Neisseria gonorrboeae. $J$ Infect Dis 128, 321-330.

Morse, S. A., Lysko, P. G., McFarland, L., Knapp, J. S., Sandstrom, E., Critchlow, C. \& Holmes, K. K. (1982). Gonococcal strains from homosexual men have outer membranes with reduced permeability to hydrophobic molecules. Infect Immun 37, 432-438.

Nikaido, H. (1994). Prevention of drug access to bacterial targets: permeability barriers and active efflux. Science 264, 382-388.

Nikaido, H. (1996). Multidrug efflux pumps of Gram-negative bacteria. J Bacteriol 178, 5853-5859.

Pan, W. \& Spratt, B. G. (1994). Regulation of the permeability of the gonococcal cell envelope by the $m$ tr system. Mol Microbiol 11, 769-775.

Paulsen, F. T., Brown, M. H. \& Skurry, R. A. (1996). Protondependent efflux systems. Microbiol Rev 60, 575-608.

Saier, M. H., Jr, Tam, R., Reizer, A. \& Reizer, J. (1994). Two novel families of bacterial membrane proteins concerned with nodulation, cell division and transport. Mol Microbiol 11, 841-847.

Sarubbi, F. A., Sparling, P. F., Blackman, E. \& Lewis, E. (1975). Loss of low-level antibiotic resistance in Neisseria gonorrhoeae due to env mutations. J Bacteriol 124, 750-756.

Shafer, W. M., Guymon, L. F., Lind, I. \& Sparling, P. F. (1984). Identification of an envelope mutation (env-10) resulting in increased antibiotic susceptibility and pyocin resistance in a clinical isolate of Neisseria gonorrhoeae. Antimicrob Agents Chemother 25, 767-769.

Shafer, W. M., Balthazar, J. T., Hagman, K. E. \& Morse, S. A. (1995). Missense mutations that alter the DNA-binding domain of the MtrR protein occur frequently in rectal isolates of Neisseria gonorrboeae that are resistant to faecal lipids. Microbiology 141, 907-911.

Sparling, P. F., Sarubbi, F. A. \& Blackman, E. (1975). Inheritance of low-level resistance to penicillin, tetracycline, and chloramphenicol in Neisseria gonorrboeae. J Bacteriol 124, 740-749.

Received 30 September 1997; accepted 19 November 1997. 